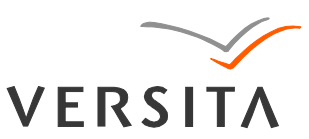

GEOCHRONOMETRIA 38(2) 2011: 116-127

DOI 10.2478/s13386-011-0012-y

Available online at

www.springerlink.com

\title{
THE NORTH DVINA RIVER DELTA DEVELOPMENT OVER THE HOLOCENE: GEOCHRONOLOGY AND PALAEOENVIRONMENT
}

\author{
NATALIYA E. ZARETSKAYA ${ }^{1}$, NATALIYA V. SHEVCHENKO ${ }^{2}$, ALEXANDRA N. SIMAKOVA ${ }^{1}$ \\ and LEOPOLD D. SULERZHITSKY ${ }^{1}$ \\ ${ }^{I}$ Geological Institute of Russian Academy of Sciences, Pyzhevsky per., 7, Moscow, 119017, Russia \\ ${ }^{2}$ Geography Faculty, Moscow State University n.a. M.V. Lomonosov, Vorobiovy Gory, Moscow, 119992, Russia
}

Received 7 June 2010

Accepted 26 October 2010

\begin{abstract}
In this paper, a detailed overview of the Holocene evolution of the North Dvina river (ND) delta (southern White Sea) is presented; it is based on radiocarbon dating, geomorphological and other field surveys, and plant macrofossil and palynological data. We have identified three main stages of the delta evolution: estuary erosional (Allerød - $5700 \mathrm{cal} \mathrm{BC}$ ), lagoon or tidal-marsh (5700 cal BC $-3700 \mathrm{cal} \mathrm{BC})$ and fan-delta accumulative $(3700 \mathrm{cal} \mathrm{BC}$ - present). These stages are correlated with local climatic curves, sea level changes, glacioisostatic raise curve and Baltic Sea stages. A variety of landforms has been identified and dated within the delta. These results help to explain the spatial and temporal patterns in the prehistoric human occupation of this area.
\end{abstract}

Keywords: North Dvina River delta, White Sea, Holocene, geochronology, radiocarbon dating, landforms, palaeoenvironmental reconstructions, land evolution.

\section{INTRODUCTION}

Large deltas entering cold tidal seas provide an opportunity to reconstruct the development of frontier landforms over time. This is an area of fluvial-marine interaction, with mosaic landscape and specific organic-rich sediments and sometimes deltas became inhabited by prehistoric people communities. Therefore deltas provide detailed archives for the studies of chronology of various palaeoenvironmental changes such as sea level oscillations, river dynamics, shoreline evolution, peat bog development, people adaptation etc.

Since 2005, we performed a detailed radiocarbon dating and palaeoenvironmental studies of the North Dvina (ND) river delta and adjacent areas (Zaretskaya, 2006; Zaretskaya et al., 2008; 2009). The main goal of this research was to reconstruct the Holocene chronology and

Corresponding author: N. E. Zaretskaya

e-mail: n_zaretskaya@inbox.ru palaeoenvironmental history of the ND delta as a case study of such a landscape evolution, and as an area of ancient people settling.

\section{STUDY AREA}

The ND delta is located in the northeastern part of Europe where the North Dvina river enters the White Sea (Fig. 1A). Nowadays this is a lobe-type fan delta with many river arms and islands. Its surface area is $1100 \mathrm{~km}^{2}$, the maximum length $-45 \mathrm{~km}$ (Lupachev, 1984) and its maximum elevation is 6-7 $\mathrm{m}$ a.s.l. Two big cities with well developed transport system - Arkhangelsk in the east and Severodvinsk in the west - make it easier to access the study area by truck, bus or regular boat.

During LGM (18-16 kyr BP) the White Sea was covered by ca. $2 \mathrm{~km}$ thick ice (Demidov et al., 2006). There is a hypothesis of an ice-dammed lake affecting this area during the deglaciation period (Bølling-Allerød) (Demi- 


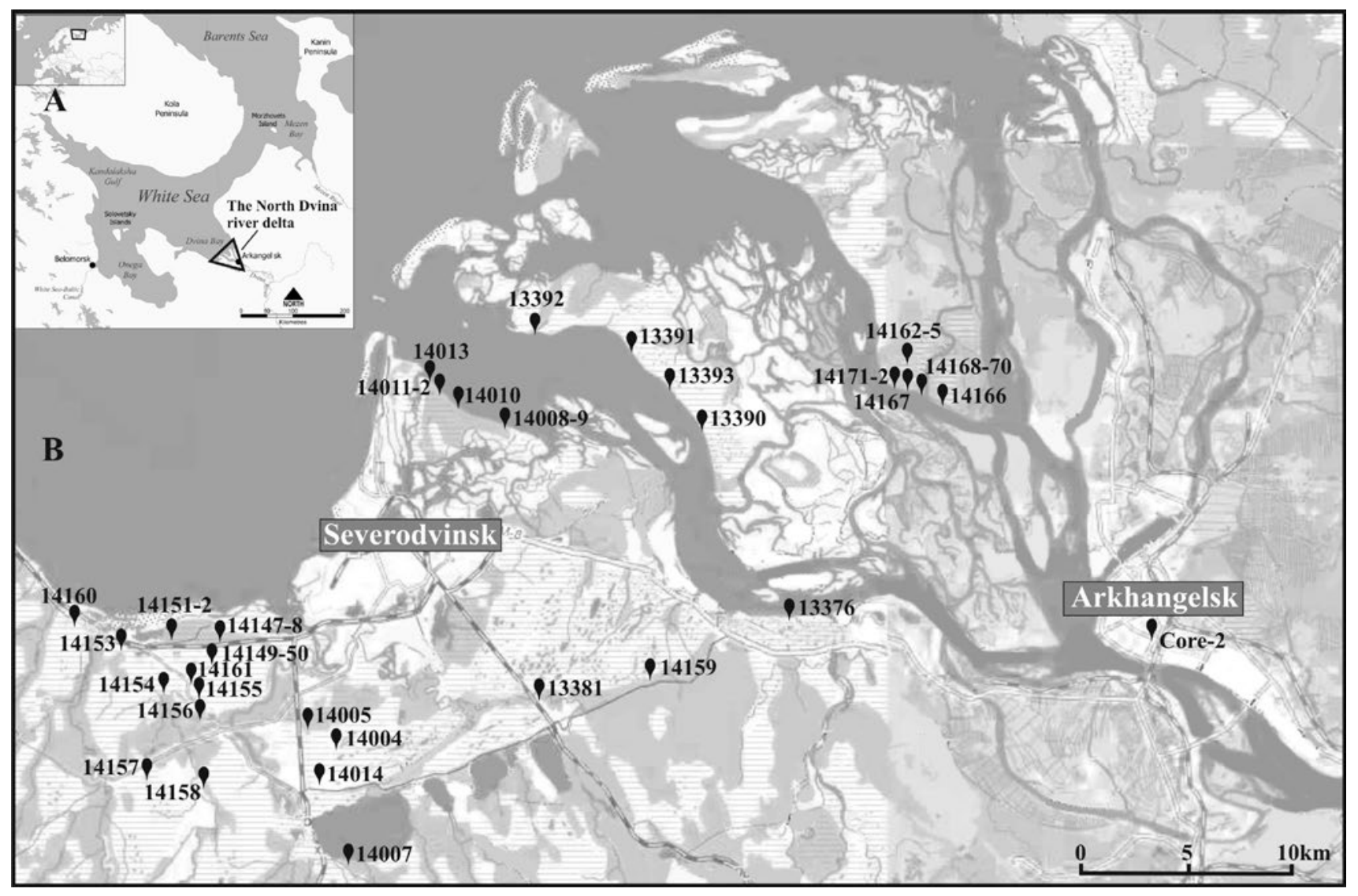

Fig. 1. Study area: the North Dvina River delta on a map (A) and sampling sites with GIN-laboratory numbers on the ND delta chart (B).

dov et al., 2006; Lavrov and Potapenko, 2005), though in the field we still could not see any traces or deposits of such a lake. The time of complete deglaciation of the White Sea and the opening of the White Sea Gorge (Gorlo in Russian, a strait connecting the White and Barents seas) is still not well known (Demidov et al., 2006). Palynological and diatom data obtained in 1960s indicates the presence of marine diatoms in the Allerød sediments within the ND delta (Pleshivtseva, 1977).

A tectonic pattern of the North Dvina delta area is still polemical. There are two controversial theories:

1) The ND delta is located within the southeastern end of the huge Kandalaksha-Dvina rift, which runs in the NW-SE direction from Kandalaksha inlet across the central part of the White Sea to Dvinsky inlet. This rift has been experiencing continuous subsidence during the Holocene (Zhivotovskaya, 1960; Avenarius, 2004). The flanks of this rift are, per contra, rising due to the glacioisostatic uplift. This theory was based on morphostructural data (Avenarius, 2004) and a finding of the Early Holocene buried peat in a borehole in Arkhangelsk city at the depth of 17.5 m (Zhivotovskaya, 1960; Nikonov, 1977; Koshechkin, 1979).

2) This is an area of recent continuous uplift due to the rise of the Arkhangelsk swell of crystalline basement
(Zykov et al., 2008). Facts which support this theory are 1) pattern of Quaternary deposits in this area; 2) North Dvina river channel pattern (a bend marking a border of the swell).

From our point of view, this contradiction between these two hypotheses is caused by a lack of geological and geochronological data for this area. Our new data allows us to propose another type of tectonic movements within this area.

\section{METHODS}

The main method was the radiocarbon dating of peat bogs of different origin, composition, depth and size, widely spread over the delta and adjacent areas. We have dated more than 50 samples of peat, gyttja, loamy peat (lagoon deposits), and wood (Table 1) collected from 30 sampling sites all over the area (Fig. 1B). The stratigraphy of relatively dry peat bogs was studied in the excavations and outcrops; wet mires were sampled with the help of the Russian corer TBG-1 (Figs. 4 and 5).

Radiocarbon dating was performed in the conventional ${ }^{14} \mathrm{C}$ laboratory within the Laboratory of Isotope Geochemistry and Geochronology in the Geological Institute of Russian Academy of Sciences (Moscow), using the standard methods of pretreatment and calculations 
Table 1. Radiocarbon dates obtained within the North Dvina River delta and the adjacent areas, with plant composition of dated samples and calibrated age ranges; Dates TIn-125 and T/n-126 from Koshechkin et al., 1977.

\begin{tabular}{|c|c|c|c|c|c|c|c|c|}
\hline No & Field number & $\begin{array}{l}\text { Lab (GIN) } \\
\text { code }\end{array}$ & $\begin{array}{l}\text { Geographical } \\
\text { coordinates } \\
\left(^{\circ}\right)\end{array}$ & $\begin{array}{l}\text { Dated organic } \\
\text { matter (with plant } \\
\text { composition) }\end{array}$ & Depth (m) & ${ }^{14} \mathrm{C}$ date & $\begin{array}{c}\text { Calibrated age range } \\
(1 \sigma) \\
(68.2 \%)\end{array}$ & $\begin{array}{c}\text { Calibrated age range } \\
(2 \sigma) \\
(95.4 \%)\end{array}$ \\
\hline 1 & - & TIn-126 & & buried peat & 17.70 & $9115 \pm 100$ & $8470-8240 \mathrm{BC}(68.2 \%)$ & $8650-7950 \mathrm{BC}(95.4 \%)$ \\
\hline 2 & SD0933/A1 & 14158 & \begin{tabular}{|l} 
N64.47188 \\
E39.64941 \\
\end{tabular} & gyttja & $3.30-3.40$ & $9070 \pm 60$ & $8320-8230 \mathrm{BC}(68.2 \%)$ & $\begin{array}{c}8470-8200 \mathrm{BC}(94.1 \%) \\
8040-8010 \mathrm{BC}(1.3 \%)\end{array}$ \\
\hline 3 & SD0941/A4 & 14165 & $\begin{array}{l}\text { N64.64880 } \\
\text { E40.32576 }\end{array}$ & loamy peat & $4.70-4.80$ & $9030 \pm 70$ & $\begin{array}{c}8310-8200 \mathrm{BC}(60.2 \%) \\
8110-8090 \mathrm{BC}(2.3 \%) \\
8040-8010 \mathrm{BC}(5.8 \%)\end{array}$ & $\begin{array}{c}8430-8360 \mathrm{BC}(2.6 \%) \\
8350-7960 \mathrm{BC}(92.8 \%)\end{array}$ \\
\hline 4 & - & TIn-125 & & buried peat & 17.25 & $8370 \pm 170$ & 7550 - 7310BC (68.2\%) & $7590-7170 \mathrm{BC}(95.4 \%)$ \\
\hline 5 & SD0807/A2 & 14007 & $\begin{array}{l}\text { N64.44381 } \\
\text { E39.78779 }\end{array}$ & peat & $2.10-2.25$ & $8610 \pm 60$ & 7710 - $7570 \mathrm{BC}(68.2 \%)$ & 7760 - $7530 \mathrm{BC}(95.4 \%)$ \\
\hline 6 & SD0941/A3 & 14164 & $\begin{array}{l}\text { N64.64880 } \\
\text { E40.32576 }\end{array}$ & moss-wood peat & $4.20-4.30$ & $7950 \pm 50$ & $\begin{array}{c}7030-6930 \mathrm{BC}(26.8 \%) \\
6920-6870 \mathrm{BC}(12.6 \%) \\
6850-6750 \mathrm{BC}(26.9 \%) \\
6720-6710 \mathrm{BC}(2.0 \%)\end{array}$ & 7050 - 6690BC (95.4\%) \\
\hline 7 & SD0943/A1 & 14167 & \begin{tabular}{|l|} 
N64.63770 \\
E40.32891
\end{tabular} & $\begin{array}{l}\text { Phragmites loamy } \\
\text { peat }\end{array}$ & $1.34-1.36$ & $6920 \pm 40$ & $5840-5745 \mathrm{BC}(68.2 \%)$ & $5890-5720 \mathrm{BC}(95.4 \%)$ \\
\hline 8 & RIKAS3/A14 & $13376 m$ & $\begin{array}{l}\mathrm{N} 64.54607 \\
\mathrm{E} 40.22030\end{array}$ & $\begin{array}{l}\text { Phragmites-repleted } \\
\text { lagoon deposits with } \\
\text { saltish water diatoms }\end{array}$ & 3.50 & $6400 \pm 30$ & $\begin{array}{c}5470-5440 \mathrm{BC}(16.8 \%) \\
5430-5400 \mathrm{BC}(9.5 \%) \\
5380-5320 \mathrm{BC}(42.0 \%)\end{array}$ & $5470-5320 \mathrm{BC}(95.4 \%)$ \\
\hline 9 & SD0945/A2 & 14170 & \begin{tabular}{|l|} 
N64.63778 \\
E40.33356 \\
\end{tabular} & $\begin{array}{l}\text { Phragmites-repleted } \\
\text { loam }\end{array}$ & $1.36-1.38$ & $6200 \pm 40$ & $\begin{array}{c}5220-5200 \mathrm{BC}(8.8 \%) \\
5180-5060 \mathrm{BC}(59.4 \%)\end{array}$ & $5300-5040 \mathrm{BC}(95.4 \%)$ \\
\hline 10 & SD0944/A1 & 14168 & \begin{tabular}{|l|}
$\mathrm{N} 64.63763$ \\
$\mathrm{E} 40.33627$ \\
\end{tabular} & plant detritus & $1.48-1.50$ & $6180 \pm 40$ & $\begin{array}{c}5210-5190 \mathrm{BC}(6.1 \%) \\
5180-5060 \mathrm{BC}(62.1 \%)\end{array}$ & $\begin{array}{c}5290-5270 \mathrm{BC}(1.4 \%) \\
5230-5000 \mathrm{BC}(94.0 \%)\end{array}$ \\
\hline 11 & RIKAS3/A14 & $13376 \mathrm{k}$ & \begin{tabular}{|l|}
$\mathrm{N} 64.54607$ \\
$\mathrm{E} 40.22030$ \\
\end{tabular} & $\begin{array}{l}\text { Phragmites-repleted } \\
\text { lagoon deposits }\end{array}$ & 3.50 & $5970 \pm 40$ & $4910-4790 \mathrm{BC}(68.2 \%)$ & $4960-4720 \mathrm{BC}(95.4 \%)$ \\
\hline 12 & SD0941/A2 & 14163 & $\begin{array}{l}\text { N64.64880 } \\
\text { E40.32576 } \\
\end{array}$ & pine-birch peat & $3.20-3.30$ & $5860 \pm 40$ & $4785-4690 \mathrm{BC}(68.2 \%)$ & $4830-4610 \mathrm{BC}(95.4 \%)$ \\
\hline 13 & SD0945/A1 & 14169 & & $\begin{array}{l}\text { Phragmites-repleted } \\
\text { loam }\end{array}$ & $0.60-0.62$ & $5440 \pm 40$ & $\begin{array}{l}4340-4310 \mathrm{BC}(26.8 \%) \\
4305-4255 \mathrm{BC}(41.4 \%)\end{array}$ & $4360-4230 \mathrm{BC}(95.4 \%)$ \\
\hline 14 & RIKAS3/A13 & 13375 & $\begin{array}{l}\text { N64.54607 } \\
\text { E40.22030 }\end{array}$ & $\begin{array}{l}\text { Phragmites loamy } \\
\text { peat (lagoon depos- } \\
\text { its) }\end{array}$ & 3.24 & $5410 \pm 40$ & $4330-4245 B C(68.2 \%)$ & $\begin{array}{c}4350-4220 \mathrm{BC}(85.2 \%) \\
4210-4160 \mathrm{BC}(6.9 \%) \\
4130-4110 \mathrm{BC}(1.0 \%) \\
4100-4070 \mathrm{BC}(2.3 \%)\end{array}$ \\
\hline 15 & SD0934/A1 & 14159 & $\begin{array}{l}\text { N64.51975 } \\
\text { E40.07372 }\end{array}$ & birch peat & $4.35-4.45$ & $4950 \pm 50$ & $3780-3660 \mathrm{BC}(68.2 \%)$ & $\begin{array}{c}3940-3870 \mathrm{BC}(7.8 \%) \\
3810-3640 \mathrm{BC}(87.6 \%)\end{array}$ \\
\hline 16 & SD0932/A1 & 14157 & \begin{tabular}{|l} 
N64.47533 \\
E39.59084
\end{tabular} & wood peat & $2.5-2.6$ & $4870 \pm 40$ & $3695-3635 \mathrm{BC}(68.2 \%)$ & $\begin{array}{c}3760-3620 \mathrm{BC}(86.3 \%) \\
3580-3530 \mathrm{BC}(9.1 \%)\end{array}$ \\
\hline 17 & KORODA/A5 & 13381 & $\begin{array}{l}\text { N64.50906 } \\
\text { E39.97263 }\end{array}$ & peat & 1.75 & $4570 \pm 30$ & $\begin{array}{l}3370-3330 \mathrm{BC}(42.5 \%) \\
3220-3180 \mathrm{BC}(14.8 \%) \\
3160-3130 \mathrm{BC}(10.9 \%)\end{array}$ & $\begin{array}{c}3500-3460 \mathrm{BC}(6.7 \%) \\
3380-3310 \mathrm{BC}(46.2 \%) \\
3240-3100 \mathrm{BC}(42.4 \%)\end{array}$ \\
\hline 18 & SD0942/A1 & 14166 & $\begin{array}{l}\text { N64.63523 } \\
\text { E40.35748 }\end{array}$ & loamy peat & $3.40-3.50$ & $4430 \pm 70$ & $\begin{array}{c}3330-3230 \mathrm{BC}(20.0 \%) \\
3170-3160 \mathrm{BC}(1.7 \%) \\
3120-2920 \mathrm{BC}(46.5 \%) \\
\end{array}$ & $3340-2910 \mathrm{BC}(95.4 \%)$ \\
\hline 19 & SD0935/A1 & 14160 & $\begin{array}{l}\text { N64.54092 } \\
\text { E39.53151 }\end{array}$ & $\begin{array}{l}\text { loamy peat (lagoon } \\
\text { deposits) with } \\
\text { Phragmites and } \\
\text { saltish water diatoms }\end{array}$ & $4.20-4.30$ & $4360 \pm 60$ & $\begin{array}{c}3090-3060 \mathrm{BC}(6.5 \%) \\
3030-2900 \mathrm{BC}(61.7 \%)\end{array}$ & $\begin{array}{c}3330-3220 \mathrm{BC}(7.2 \%) \\
3120-2880 \mathrm{BC}(88.2 \%)\end{array}$ \\
\hline 20 & LASOM/A9 & 13390 & $\begin{array}{l}\text { N64.62278 } \\
\text { E40.12851 }\end{array}$ & $\begin{array}{l}\text { loamy peat (lagoon } \\
\text { deposits) with saltish } \\
\text { water diatoms }\end{array}$ & 2.25 & $4060 \pm 50$ & $\begin{array}{c}2840-2810 \mathrm{BC}(6.4 \%) \\
2670-2490 \mathrm{BC}(61.8 \%)\end{array}$ & $\begin{array}{l}2860-2800 \mathrm{BC}(12.4 \%) \\
2760-2470 \mathrm{BC}(83.0 \%)\end{array}$ \\
\hline 21 & RIKAS3/A12 & 13374 & $\begin{array}{l}\text { N64.54607 } \\
\text { E40.22030 }\end{array}$ & $\begin{array}{l}\text { Phragmites-birch } \\
\text { loamy peat with } \\
\text { saltish water diatoms }\end{array}$ & $2.57-2.59$ & $3880 \pm 40$ & $2460-2290 \mathrm{BC}(68.2 \%)$ & $\begin{array}{c}2470-2270 \mathrm{BC}(88.2 \%) \\
2260-2200 \mathrm{BC}(7.2 \%)\end{array}$ \\
\hline 22 & SD0822/A1 & 14014 & $\begin{array}{l}\text { N64.47707 } \\
\text { E39.77145 }\end{array}$ & $\begin{array}{l}\text { loamy peat (lagoon } \\
\text { deposits) }\end{array}$ & $2.20-2.30$ & $3530 \pm 40$ & $\begin{array}{l}1930-1860 \mathrm{BC}(29.9 \%) \\
1850-1770 \mathrm{BC}(38.3 \%)\end{array}$ & $1970-1740 \mathrm{BC}(95.4 \%)$ \\
\hline 23 & SD0932/A1 & 14156 & \begin{tabular}{|l} 
N64.47533 \\
E39.59084
\end{tabular} & Sphagnum peat & $1.55-1.65$ & $3490 \pm 40$ & $1880-1750 \mathrm{BC}(68.2 \%)$ & $\begin{array}{c}1920-1730 \mathrm{BC}(91.5 \%) \\
1720-1690 \mathrm{BC}(3.9 \%)\end{array}$ \\
\hline 24 & SD0929/A1 & 14155 & $\begin{array}{l}\text { N64.50837 } \\
\text { E39.62666 } \\
\end{array}$ & Phragmites peat & $1.95-2.00$ & $3370 \pm 40$ & $\begin{array}{l}1740-1710 \mathrm{BC}(12.9 \%) \\
1700-1610 \mathrm{BC}(55.3 \%)\end{array}$ & $1750-1530 \mathrm{BC}(95.4 \%)$ \\
\hline 25 & RIKAS3/A10 & 13372 & $\begin{array}{l}\text { N64.54607 } \\
\text { E40.22030 } \\
\end{array}$ & $\begin{array}{l}\text { Sphagnum with green } \\
\text { mosses peat }\end{array}$ & 2.11-2.12 & $3200 \pm 40$ & $1505-1430 \mathrm{BC}(68.2 \%)$ & $\begin{array}{c}1610-1570 \mathrm{BC}(2.7 \%) \\
1540-1390 \mathrm{BC}(92.7 \%)\end{array}$ \\
\hline 26 & SD0946/A2 & 14172 & \begin{tabular}{|l|} 
N64.63838 \\
E40.32116 \\
\end{tabular} & drift wood (twigs) & $1.00-1.17$ & $3030 \pm 60$ & $1390-1210 \mathrm{BC}(68.2 \%)$ & $1430-1110 \mathrm{BC}(95.4 \%)$ \\
\hline 27 & SD0946/A1 & 14171 & \begin{tabular}{|l} 
N64.63838 \\
E40.32116
\end{tabular} & drift wood (twigs) & $1.00-1.17$ & $3010 \pm 80$ & $1390-1120 \mathrm{BC}(68.2 \%)$ & $1430-1010 \mathrm{BC}(95.4 \%)$ \\
\hline
\end{tabular}


Table 1. Continuation

\begin{tabular}{|c|c|c|c|c|c|c|c|c|}
\hline No & Field number & $\begin{array}{l}\text { Lab (GIN) } \\
\text { code }\end{array}$ & $\begin{array}{l}\text { Geographical } \\
\text { coordinates } \\
\left(^{\circ}\right) \\
\end{array}$ & \begin{tabular}{|l|} 
Dated organic \\
matter (with plant \\
composition)
\end{tabular} & Depth (m) & ${ }^{14} \mathrm{C}$ date & $\begin{array}{c}\text { Calibrated age range } \\
(1 \sigma) \\
(68.2 \%)\end{array}$ & $\begin{array}{c}\text { Calibrated age range } \\
(2 \sigma) \\
(95.4 \%)\end{array}$ \\
\hline 28 & LASOM4 & 13393 & $\begin{array}{l}\text { N64.63567 } \\
\text { E40.10415 }\end{array}$ & $\begin{array}{l}\text { loamy peat (lagoon } \\
\text { deposit)) with saltish } \\
\text { water diatoms }\end{array}$ & 1.80 & $2990 \pm 40$ & $\begin{array}{l}1310-1190 \mathrm{BC}(55.8 \%) \\
1180-1130 \mathrm{BC}(12.4 \%)\end{array}$ & $\begin{array}{c}1390-1110 \mathrm{BC}(94.4 \%) \\
1100-1080 \mathrm{BC}(1.0 \%)\end{array}$ \\
\hline 29 & SD0924/A1 & 14154 & $\begin{array}{l}\text { N64.51318 } \\
\text { E39.64450 }\end{array}$ & \begin{tabular}{|l|} 
loamy peat (lagoon \\
deposits) with \\
Phragmites \\
\end{tabular} & $1.90-2.00$ & $2900 \pm 40$ & $\begin{array}{c}1190-1180 \mathrm{BC}(2.7 \%) \\
1160-1140 \mathrm{BC}(3.6 \%) \\
1130-1010 \mathrm{BC}(61.9 \%)\end{array}$ & $\begin{array}{l}1260-1230 \mathrm{BC}(2.7 \%) \\
1220-970 \mathrm{BC}(92.7 \%)\end{array}$ \\
\hline 30 & RIKAS3/A7 & 13369кл & $\begin{array}{l}\text { N64.54607 } \\
\text { E40.22030 }\end{array}$ & Sphagnum peat & 1.60 & $2800 \pm 80$ & $1050-840 \mathrm{BC}(68.2 \%)$ & $1200-800 \mathrm{BC}(95.4 \%)$ \\
\hline 31 & RIKAS3/A7 & 13369 & $\begin{array}{l}\text { N64.54607 } \\
\text { E40.22030 }\end{array}$ & Sphagnum peat & 1.60 & $2690 \pm 80$ & $930-790 \mathrm{BC}(68.2 \%)$ & $\begin{array}{c}1060 \text { - 740BC }(92.1 \%) \\
690 \text { - 660BC }(1.3 \%) \\
650-590 \mathrm{BC}(1.9 \%)\end{array}$ \\
\hline 32 & LASOM2 & 13391 & $\begin{array}{l}\text { N64.64983 } \\
\text { E40.06901 }\end{array}$ & $\begin{array}{l}\text { Phragmites peat with } \\
\text { saltish water diatoms }\end{array}$ & $1.08-1.10$ & $2570 \pm 40$ & $\begin{array}{c}810-750 \mathrm{BC}(49.3 \%) \\
690-660 \mathrm{BC}(11.4 \%) \\
640-590 \mathrm{BC}(7.5 \%) \\
\end{array}$ & $\begin{array}{l}820-730 \mathrm{BC}(55.2 \%) \\
690-660 \mathrm{BC}(13.8 \%) \\
650-540 \mathrm{BC}(26.4 \%)\end{array}$ \\
\hline 33 & RIKAS3/A6 & 13368 & $\begin{array}{l}\text { N64.54607 } \\
\text { E40.22030 }\end{array}$ & Sphagnum peat & $1.31-1.32$ & $2450 \pm 50$ & $\begin{array}{c}750-680 \mathrm{BC}(18.9 \%) \\
670-640 \mathrm{BC}(6.3 \%) \\
590-580 \mathrm{BC}(1.9 \%) \\
560-410 \mathrm{BC}(41.1 \%)\end{array}$ & $\begin{array}{l}770-680 \mathrm{BC}(23.0 \%) \\
670-400 \mathrm{BC}(72.4 \%)\end{array}$ \\
\hline 34 & SD0923/A1 & 14153 & $\begin{array}{l}\text { N64.53192 } \\
\text { E39.57147 }\end{array}$ & $\begin{array}{l}\text { organo-mineral gyttja } \\
\text { with saltish water } \\
\text { diatoms }\end{array}$ & $2.95-3.05$ & $2370 \pm 50$ & $520-380 \mathrm{BC}(68.2 \%)$ & $\begin{array}{c}750-680 \mathrm{BC}(10.9 \%) \\
670-630 \mathrm{BC}(3.1 \%) \\
600-360 \mathrm{BC}(81.3 \%)\end{array}$ \\
\hline 35 & SD0941/A1 & 14162 & $\begin{array}{l}\text { N64.64880 } \\
\text { E40.32576 }\end{array}$ & Sphagnum peat & $1.95-2.05$ & $2200 \pm 70$ & $370-190 \mathrm{BC}(68.2 \%)$ & $\begin{array}{c}400 \text { - 90BC }(94.3 \%) \\
70 \text { - 50BC }(1.1 \%)\end{array}$ \\
\hline 36 & SD0809/A2 & 14012 & \begin{tabular}{|l|} 
N64.63898 \\
E39.87736
\end{tabular} & $\begin{array}{l}\text { loamy peat (lagoon } \\
\text { deposits) }\end{array}$ & $0.68-0.70$ & $2200 \pm 40$ & $\begin{array}{l}360-270 \mathrm{BC}(42.2 \%) \\
260-200 \mathrm{BC}(26.0 \%)\end{array}$ & $390-170 \mathrm{BC}(95.4 \%)$ \\
\hline 37 & SD0922/A1 & 14151 & $\begin{array}{l}\text { N64.53582 } \\
\text { E39.63453 }\end{array}$ & $\begin{array}{l}\text { organo-mineral gyttja } \\
\text { (lagoon deposits) with } \\
\text { saltish water diatoms }\end{array}$ & $1.10-1.20$ & $2160 \pm 50$ & $\begin{array}{c}360-280 \mathrm{BC}(29.1 \%) \\
240-150 \mathrm{BC}(32.1 \%) \\
140-110 \mathrm{BC}(7.0 \%)\end{array}$ & $370-50 \mathrm{BC}(95.4 \%)$ \\
\hline 38 & LASOM3 & 13392 & $\begin{array}{l}\text { N64.66272 } \\
\text { E39.97818 }\end{array}$ & $\begin{array}{l}\text { loamy peat (lagoon } \\
\text { deposits) } \\
\text { with Phragmites and } \\
\text { saltish water diatoms }\end{array}$ & $1.30-1.33$ & $2030 \pm 40$ & $100-30 \mathrm{AD}(68.2 \%)$ & $170 \mathrm{BC}(95.4 \%) 60 \mathrm{AD}$ \\
\hline 39 & SD0817/A1 & 14008 & \begin{tabular}{|l|} 
N64.62661 \\
E39.92014 \\
\end{tabular} & \begin{tabular}{|l|} 
loamy peat (lagoon \\
deposits)
\end{tabular} & 1.35 & $2010 \pm 30$ & $45-25 \mathrm{AD}(68.2 \%)$ & $100 \mathrm{BC}(95.4 \%) 70 \mathrm{AD}$ \\
\hline 40 & SD0805/A1 & 14004 & \begin{tabular}{|l|} 
N64.48435 \\
E39.77351
\end{tabular} & peat & $2.10-2.20$ & $1960 \pm 50$ & $40-90 \mathrm{AD}(68.2 \%)$ & 100BC (95.4\%) 140AD \\
\hline 41 & RIKAS3/A5 & 13367 & \begin{tabular}{|l|} 
N64.54607 \\
E40.22030 \\
\end{tabular} & Sphagnum peat & $1.05-1.06$ & $1960 \pm 40$ & $\begin{array}{c}20-10 \mathrm{BC}(3.6 \%) \\
A D-90 \mathrm{AD}(64.6 \%)\end{array}$ & $50 \mathrm{BC}(95.4 \%)$ 130AD \\
\hline 42 & SD0817/A2 & 14009 & \begin{tabular}{|l} 
N64.62661 \\
E39.92014
\end{tabular} & $\begin{array}{l}\text { loamy peat (lagoon } \\
\text { deposits) }\end{array}$ & $1.62-1.65$ & $1860 \pm 40$ & $80-110 \mathrm{AD}(68.2 \%)$ & $60 \mathrm{AD}(95.4 \%) 250 \mathrm{AD}$ \\
\hline 43 & SD0812/A1 & 14010 & $\begin{array}{l}\text { N64.63369 } \\
\text { E39.89173 }\end{array}$ & peat & $1.28-1.30$ & $1820 \pm 30$ & $\begin{array}{l}135-200 A D(46.1 \%) \\
205-235 A D(22.1 \%)\end{array}$ & $\begin{array}{c}\text { 90AD (1.1\%) 100AD } \\
\text { 120AD (91.9\%) 260AD } \\
300 \mathrm{AD}(2.5 \%) 320 \mathrm{AD}\end{array}$ \\
\hline 44 & SD0937/A1 & 14161 & \begin{tabular}{|l|} 
N64.51826 \\
E39.64148 \\
\end{tabular} & \begin{tabular}{|l|} 
loamy peat (lagoon \\
deposits)
\end{tabular} & $1.70-1.80$ & $1760 \pm 70$ & $\begin{array}{c}170 \text { - 190AD (3.9\%) } \\
210-390 A D(64.3 \%)\end{array}$ & $\begin{array}{c}80 \mathrm{AD}(1.8 \%) 110 \mathrm{AD} \\
120 \mathrm{AD}(93.6 \%) 430 \mathrm{AD}\end{array}$ \\
\hline 45 & SD0806/A1 & 14005 & $\begin{array}{l}\text { N64.49657 } \\
\text { E39.74882 }\end{array}$ & peat with Phragmites & $0.90-1.00$ & $1310 \pm 40$ & $\begin{array}{l}660-720 \mathrm{AD}(48.6 \%) \\
740-770 \mathrm{AD}(19.6 \%)\end{array}$ & $640 \mathrm{AD}(95.4 \%) 780 \mathrm{AD}$ \\
\hline 46 & SD0809/A1 & 14011 & \begin{tabular}{|l|} 
N64.63898 \\
E39.87736
\end{tabular} & peat & $0.43-0.45$ & $1260 \pm 20$ & $\begin{array}{c}690-750 \mathrm{AD}(60.4 \%) \\
760-775 \mathrm{AD}(7.8 \%)\end{array}$ & $\begin{array}{l}670 \mathrm{AD}(93.5 \%) \text { 780AD } \\
\text { 790AD }(1.9 \%) 810 \mathrm{AD}\end{array}$ \\
\hline 47 & RIKAS3/A4 & 13366 & $\begin{array}{l}\text { N64.54607 } \\
\text { E40.22030 }\end{array}$ & Sphagnum peat & $0.52-0.54$ & $1080 \pm 60$ & $890-1020 \mathrm{AD}(68.2 \%)$ & $770 \mathrm{AD}(95.4 \%)$ 1050AD \\
\hline 48 & SD0821/A1 & 14013 & \begin{tabular}{|l|} 
N64.64246 \\
E39.86846
\end{tabular} & $\begin{array}{l}\text { loamy peat (lagoon } \\
\text { deposits) }\end{array}$ & $0.53-0.55$ & $1080 \pm 40$ & $\begin{array}{c}890-920 \mathrm{AD}(17.2 \%) \\
940-1020 \mathrm{AD}(51.0 \%)\end{array}$ & $880 \mathrm{AD}(95.4 \%)$ 1030AD \\
\hline 49 & SD0922/A2 & 14152 & $\begin{array}{l}\text { N64.53582 } \\
\text { E39.63453 }\end{array}$ & $\begin{array}{l}\text { organo-mineral gyttja } \\
\text { with saltish water } \\
\text { diatoms }\end{array}$ & $0.95-1.00$ & $1050 \pm 70$ & $890-1040 \mathrm{AD}(68.2 \%)$ & $810 \mathrm{AD}(95.4 \%)$ 1160AD \\
\hline 50 & SD0918/A1 & 14149 & \begin{tabular}{|l|} 
N64.52633 \\
E39.65280 \\
\end{tabular} & Carex peat & $1.00-1.10$ & $1000 \pm 60$ & $\begin{array}{c}980-1060 \mathrm{AD}(39.7 \%) \\
1080-1160 \mathrm{AD}(28.5 \%)\end{array}$ & $890 \mathrm{AD}(95.4 \%)$ 1180AD \\
\hline 51 & SD0921/A1 & 14150 & \begin{tabular}{|l|} 
N64.52959 \\
E39.65491 \\
\end{tabular} & Carex peat & $1.20-1.30$ & $970 \pm 70$ & $1010-1160 \mathrm{AD}(68.2 \%)$ & $\begin{array}{c}\text { 890AD (2.0\%) 920AD } \\
950 \mathrm{AD}(93.4 \%) \text { 1220AD }\end{array}$ \\
\hline 52 & SD0910/A1 & 14148 & \begin{tabular}{|l|} 
N64.53463 \\
E39.65773 \\
\end{tabular} & Carex peat & $0.75-0.85$ & $640 \pm 80$ & $\begin{array}{l}1280-1330 \mathrm{AD}(30.5 \%) \\
1340-1400 \mathrm{AD}(37.7 \%)\end{array}$ & $\begin{array}{c}\text { 1220AD (95.4\%) } \\
1440 \mathrm{AD} \\
\end{array}$ \\
\hline 53 & SD0909/A1 & 14147 & \begin{tabular}{|l|} 
N64.53580 \\
E39.65676 \\
\end{tabular} & wood peat & $0.75-0.85$ & $190 \pm 150$ & $1630-1960 \mathrm{AD}(68.2 \%)$ & $\begin{array}{c}\text { 1470AD (95.4\%) } \\
\text { 1960AD }\end{array}$ \\
\hline 54 & RIKAS3/A1 & 13364 & $\begin{array}{l}\text { N64.54607 } \\
\text { E40.22030 }\end{array}$ & shrub turf & $0.11-0.13$ & Modern & & \\
\hline
\end{tabular}


(Zaretskaya et al., 2001; 2007). For calibration, we used the OxCal program v.3.10 (Bronk Ramsey, 1995; 2000) and calibration curve IntCal04 (Reimer et al., 2004).

Our geomorphological studies were based on the analysis of the 1:100,000 topographic maps (Aerogeodezia, 2002), the 1:1,000,000 map of Quaternary deposits compiled by Lavrov and Potapenko (Lavrov and Potapenko, 2005) and space images (from Google Earth site). In the field, we mapped various landforms that have allowed us to compile a sketch-map of the North Dvina delta and adjacent areas (Fig. 2A). We studied spatial distribution, morphometry and deposits of these landforms. Our conclusions on the origin, age, peculiarities of evolution of various types of landforms and character of landscape-forming processes are based on the field data, GPS navigation results (World Geodetic System 1984) and stratigraphy and ${ }^{14} \mathrm{C}$ ages of deposits composing the ND delta and the adjacent areas.

For reconstructions of palaeoenvironment, we used the results of both plant macrofossil and spore-pollen analyses. Plant macrofossil analysis of all the dated samples was performed by Olga N. Uspenskaya from the Institute of Horticulture at the Russian Academy of Agri- cultural Sciences, and provided not only the plant composition data of peat and gyttja samples, but also diatom species showing salt- or freshwater conditions, and the organic/mineral ratio of the sample (Table 1). This allowed us to reconstruct the scenario of sedimentation, plant assemblages and ecology, and salinity for lagoon deposits.

Spore-pollen analysis of two sections (Rikasiha $3.5 \mathrm{~m}$ depth, and Krugly mokh $-5 \mathrm{~m}$ depth) has been performed by Alexandra N. Simakova in the Geological Institute of Russian Academy of Sciences, using the standard methods of analysis and diagram construction (Fig. 3) (Bohncke and Simakova, 2008).

\section{RESULTS}

Actually, the North Dvina delta is heterogeneous in landforms and their age, including such a various types as moraine remains on the Nikolsky Island, LiasominUglomin Island system composed of beach ridges and palaeolagoons between them, and lowland flat sandy islands of modern age and fluvial origin.

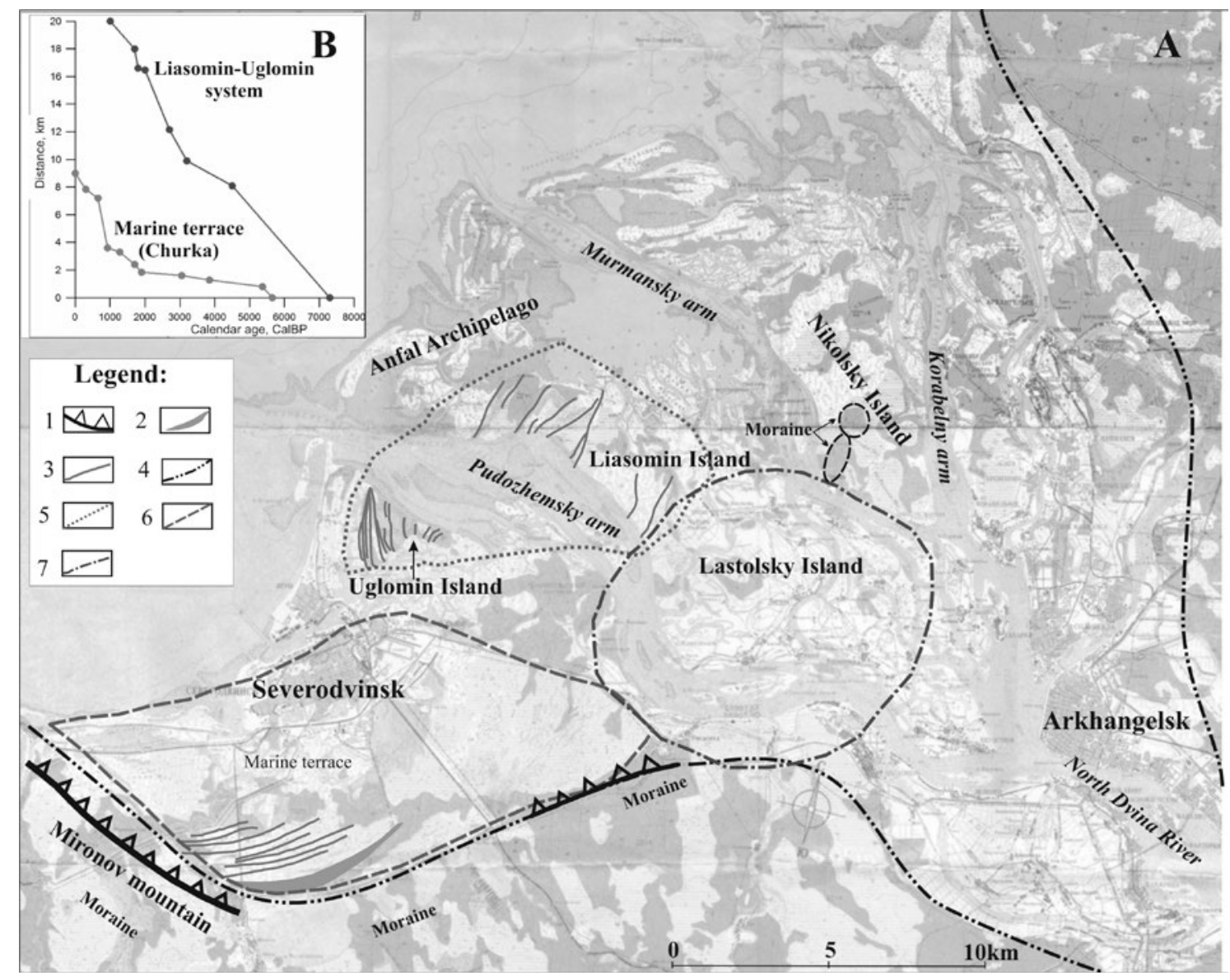

Fig. 2. (A) A sketch geomorphological map of the North Dvina River delta: 1 - Mironov mountain palaeocliff; 2 - accumulative sand body (the oldest beach ridge) in the inner part of marine terrace; 3 - beach ridges forming the terrace and islands; 4 - borders of the delta; 5 - Liasomin-Uglomin system; 6 - marine terrace (Churka site); 7 - proposed area of lagoon extension. (B) - rates of prograding of ND delta into the White Sea. 


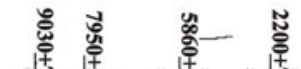

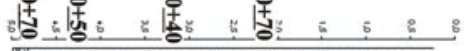

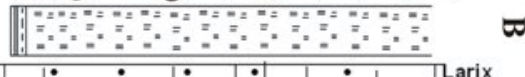

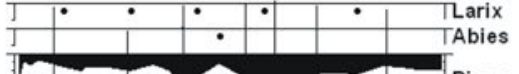

Picea

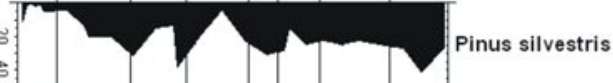

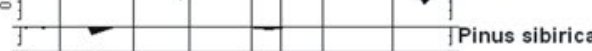

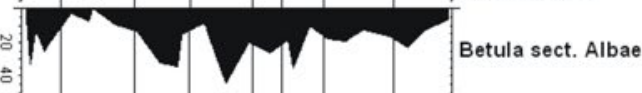

B
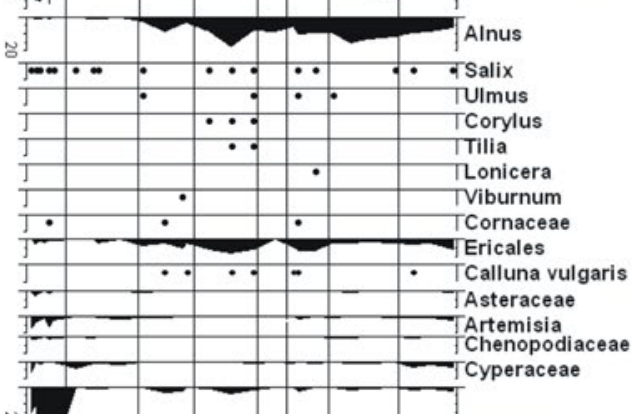

\%

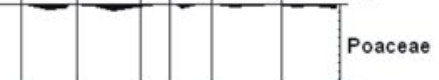

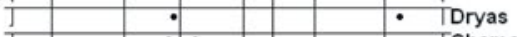

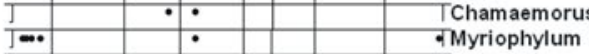

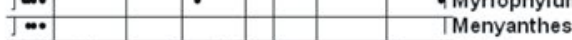

\begin{tabular}{l|l|l|l|l|l}
\hline$\bullet-\bullet$ & $\bullet$ & $\bullet$ & $\bullet$ & & $\bullet$ TTypha \\
\hline
\end{tabular}

Tÿcopodium clavatum

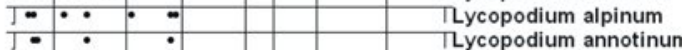

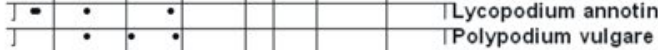

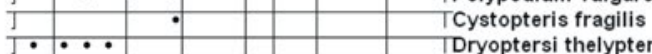

I Dryoptersi thelypteris

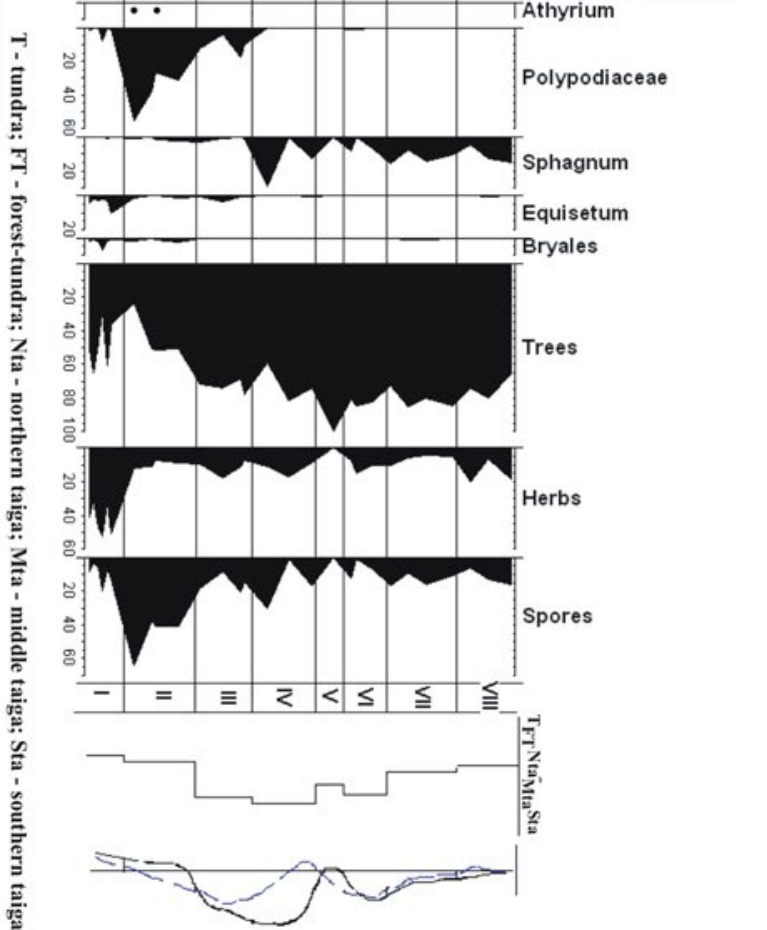

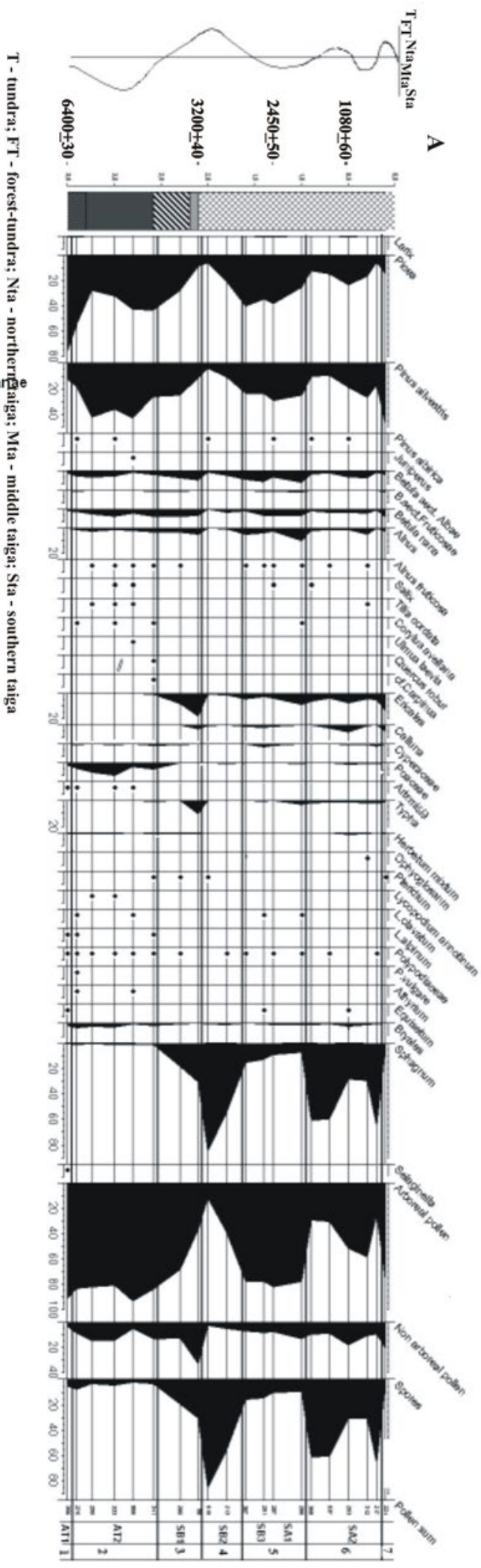




\section{Landform types}

Within the ND delta and adjacent areas, three main types of Late Pleistocene-Holocene landforms were distinguished: Late Pleistocene moraine, Holocene marine and fan-delta landforms.

Late Pleistocene landforms are presented mostly by moraine - hummocky uplands alternating with depressions, partially flattened by sea activity, with a $20-\mathrm{m}$ high scarp steeply dropping to the White Sea shore (Fig. 2B). The moraine is widespread over the delta surroundings
(50-60 m a.s.1.) and sometimes within it ("inselberg" moraine 8-9 m a.s.l. on the Nikolsky Island). Depressions on the moraine surface are mostly occupied by Holocene peat bogs of varying thickness (Fig. 4), and are submeridionally elongated. Moraine is composed of diamicton - hard clay-loam with gravel and boulders.

Other landforms and deposits are of the Holocene age. Landforms are presented mostly by series of sandy beach ridges within the western part of the delta, and by a $20 \mathrm{~m}$ high palaeocliff (Fig. 2A). This palaeocliff bordering the

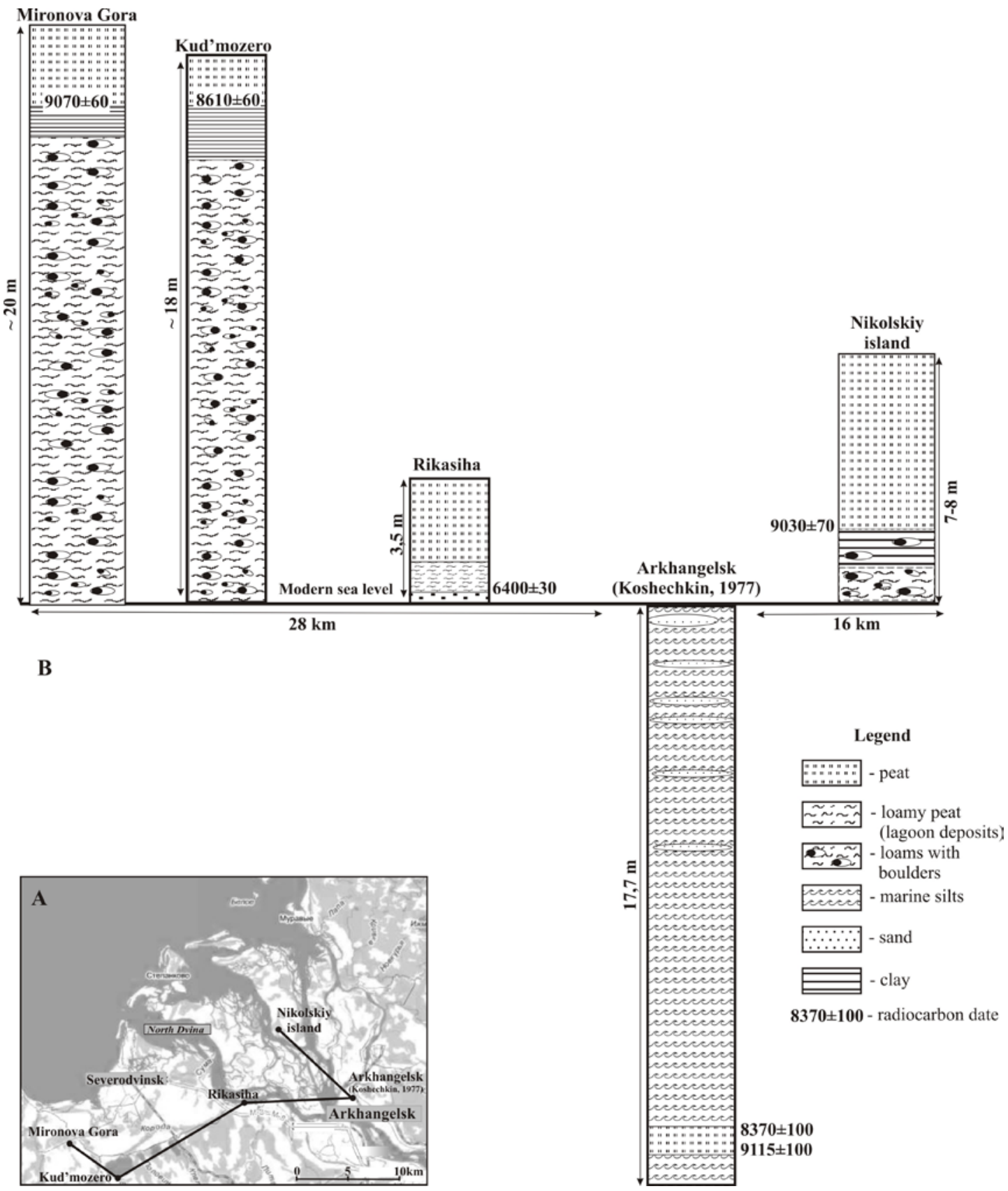

Fig. 4. A site profile illustrating location of synchronous early Holocene dates at different depths and topographic levels: $A$ - on the map; $B$ - on the sections (with depths and distances). 
delta in the south, and the biggest beach ridge are the oldest Holocene landforms within the study area. Seawards there is a marine terrace $(20-25 \mathrm{~km}$ long, 10-12 km wide and 5-8 m a.s.l.) composed of a series of younger sub-parallel beach ridges (Fig. 2A) alternating with narrow and shallow bogs. Prograding of this terrace into the White Sea occurred in the NW direction.

Deposits composing these landforms are of different origin and structure. Marine transgression sediments are represented by blue-gray solid wet clays and loams, sometimes with sand, sometimes with pebbles and boulders (when coastal erosion had been reworking moraine in the course of transgressions). They occur both inside and outside the delta, overlying moraines in former White Sea ingressive inlets, and underlying lagoon deposits within the ND river mouth. Lagoon deposits are represented by stinking grayish or brownish loams or gyttja with organic matter (remnants of Phragmites roots etc.), usually underlying mire sediments (Fig. 5). Mires and composing deposits (peat of various composition and preservation rate) are particularly widespread over the delta and adjacent areas. These are continuous vast and deep swamps overlying moraines and marine clays, or long, tight and shallow bogs between beach ridges, sometimes overlying lagoon deposits on the Liasomin and
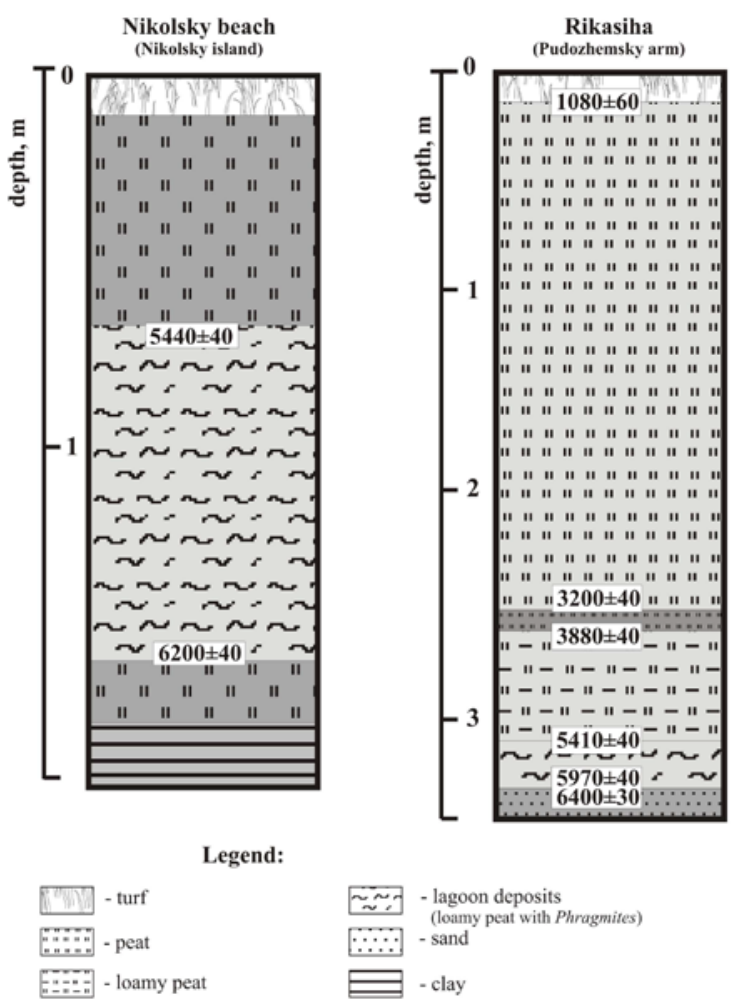

Legend:

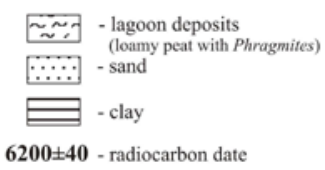

Fig. 5. Examples illustrating the lagoon deposits (loamy peat with Phragmites communis) presence in the sections within the North Dvina delta.
Uglomin islands, and directly lying on the marine sands on the terrace. Beach ridges and dunes which sometimes formed upon them are composed of fine to coarse beach sand.

All these landforms are influenced by modern erosional processes, incised by plenty of rills, temporal streams and arms. The eastern part of the marine terrace was strongly modified by human activity in 1930s while construction of the Severodvinsk (Molotovsk) city and special channels for submarine exit from the docks.

\section{Radiocarbon dates}

To reconstruct a time record and sequences of processes forming the ND delta we used radiocarbon dates obtained on samples related to all types of analyzed landforms and deposits. All dates with sample composition and calibrated age ranges are presented in the Table 1. To work with such a huge radiocarbon data set, we used "Sum probability distribution" operation from OxCal 3.10 program (Bronk Ramsey, 1995; 2000; Reimer et al., 2004); we consider that the obtained diagram can reflect the stages of the ND delta development, separating various chronological phases and periods. In the analysis, we did not include dates obtained from continuous core profiles because they do not reflect real stages of the whole area development (Fig. 6B).

\section{Palynological data}

Spore-pollen analysis of two peat cores (Rikasiha and Krugly moh) resulted in the reconstruction of consecutive transition of plant communities over the Holocene within the North Dvina delta (Fig. 3).

In the early Holocene (ca. $9000{ }^{14} \mathrm{C}$ BP, $8300-8200$ cal $\mathrm{BC}$ ), forest-tundra swampy landscapes predominated over this area, with relatively dry and cool climate.

At the end of Boreal period, sparse coniferous-birch forests and eutrophic bogs formed the landscapes, which is characteristic for the first postglacial transgression time in this area (Pleshivtseva, 1977).

At the beginning of the Atlantic period (ca. $7950{ }^{14} \mathrm{C}$ BP, 7000-6700 cal BC), forest landscapes expanded. Spruce-pine communities predominated, and they had been transforming into pine-spruce-birch complexes with rare broadleaf species and alder during the Holocene climatic optimum. In the late Atlantic optimum (ca. 5860 ${ }^{14} \mathrm{C}$ BP, 4800-4700 cal BC), a maximum of broadleaf pollen is fixed, which is well correlated with spore-pollen data from South Scandinavia (Bradshaw et al., 2000), Kola Peninsula (Lebedeva, 1977; Kremenetsky et al, 1998), Pechora lowland (Bratuschak, 2005), Onega inlet shore (Koshechkin et al., 1977) and North Dvina depression (Pleshivtseva, 1977; Baranovskaya et al., 1977).

At the beginning of Subboreal (ca. $3200{ }^{14} \mathrm{C}$ BP, 1500-1400 cal BC), forest-tundra landscapes (sparse pine-spruce-birch forests with tundra communities) began to predominate. At ca. $3000{ }^{14} \mathrm{C}$ BP $(1300-1200 \mathrm{cal} \mathrm{BC})$ 
Sphagnum peat bogs expanded over the area, adjacent areas and Kola Peninsula (Lebedeva, 1977; Kremenetsky et al, 1998; Velichko et al., 1994). In the late Subboreal (ca. $2200{ }^{14} \mathrm{C}$ BP, $400-200$ cal BC), north taiga vegetation replaced forest-tundra landscapes. Peaks of Sphagnum bogs and Ericaceae occurred within the middle subboreal and in the second half of Subatlantic time.

At the beginning of the Subatlantic time, north-taiga spruce-pine forest communities began to predominate, with some broad-leaf species. At the second half of the Subatlantic, they transformed into pine-larch and pinespruce forests alternating with tundra communities, and at the end of Subatlantic time - into pine forests.

\section{DISCUSSION: RECONSTRUCTION}

According to our data, we can distinguish three stages of the North Dvina river mouth development over the Holocene: 1) "estuary" stage (Late Glacial age - early Holocene), 2) "lagoon" stage (middle Holocene), and 3) "fan delta" stage (second half of the Holocene - till now). For more convenient presentation of our reconstruction, we used the Baltic Sea Holocene stages adapted to Barents and White Seas (Koshechkin, 1979; Kaplin and Selivanov, 1999) (Fig. 6A). In the following text, we mostly cite calibrated ages; for correlation of radiocarbon dates and calibrated ages please see Table 1 and Fig. 6.

\section{Estuary stage}

Based on published data (Demidov et al., 2006; Pleshivtseva, 1977) and our field observations we suppose that the start of estuary stage took place during the deglaciation in Allerød. At that time, the North Dvina river mouth and adjacent areas represented a moraine flatplain; we did not find any evidence indicating the existence of an ice-dammed lake within this area 13-11 kyr BP (Demidov et al., 2006; Lavrov and Potapenko, 2005).

\section{Late Glacial age}

When the White Sea Gorge (Gorlo straight) became free of ice and the water exchange between Barents and White Seas established, the White Sea level became equal with the level of the World Ocean ca. $120 \mathrm{~m}$ below the modern shoreline (Koshechkin, 1979). Hence, the erosion base level of the North Dvina River was ca. 100-120 m lower than the modern one and glacioisostatic rise of Scandinavian shield started, so we suppose a huge and deep (even catastrophic) incision of the North Dvina into the moraines, and after the early Holocene White Sea transgression and regression - into the marine deposits. The depth of this incision could exceed $50 \mathrm{~m}$ (according to the difference in absolute heights between moraine surface and bottom of Holocene deposits in the core \#2 (Koshechkin, 1979) in Arkhangelsk (Fig. 4).

\section{Portlandia-Littorina transgression (Younger Dryas - Preboreal)}

We have no dates for this period, and our conclusions are based on morphostratigraphy, lithological data and image analysis.

When the Portlandia transgression started ca. 11,000 ${ }^{14} \mathrm{C}$ BP (Kaplin and Selivanov, 1999; Fig. 6A), the primary glacial landscape of the North Dvina river mouth and adjacent areas got drowned. Ingression inlets formed along, and marine deposits (blue-gray clays) accumulated in moraine depressions, and the heights (uplands) had been smoothing: we usually found marine blue-gray clays underlying peat deposits when drilling bogs southwards of the modern ND delta (Fig. 4B), and there is evidence of marine clays and loams with special fauna under the buried peat in the Arkhangelsk core (Koshechkin, 1979). A huge estuary-type inlet existed at the place of the modern delta, with dissected shoreline and numerous islands.

\section{Littorina regression (Boreal): 8470-7310 cal BC}

For this period, we have radiocarbon dates, and sporepollen and palaeobotanic data (Table 1; Figs. 4B and 6).

Two radiocarbon dates have been earlier obtained on buried peat from the Arkhangelsk core (Koshechkin et al., 1977): $9115 \pm 100$ (Tln-126, 8240-8470 cal BC) and $8370 \pm 170$ (Tln-125, 7310-7550 cal BC) at the depth of $17.5 \mathrm{~m}$. Other three dates: $9070 \pm 60(\mathrm{GIN}-14158,8230$ 8320 cal BC) $9030 \pm 70$ (GIN-14165, 8010-8040, 8090$8110,8200-8310$ cal BC), 8610 \pm 60 (GIN-14007, 75007710 cal BC) have been received for our samples originating from core bottoms in the mires westwards and southwards from the modern delta (Mironov mountain and Kud'mozero), and on the Nikolsky Island within it (Table 1 and Fig. 4A). All the dated mires are located within the LGM moraine depressions - palaeoinlets of the Portlandia-Littorina Sea.

As it is well seen in the profile (Fig. 4B), the subsynchronous dates are located at different depths, and, consequently, at different levels relative to the modern sea level. We have the following explanation of this phenomenon: as it was considered above, before the first Holocene White Sea transgression, a deep North Dvina river incision took place. After the start of Portlandia transgression, this part of the river bed was being filled by marine deposits, and synchronously marine clays deposited within the ingression inlets. During the Littorina regressive stage, the North Dvina river was incising again, and we suppose that overgrowing oxbow lakes in abandoned palaeochannels could have formed within its valley, and on the moraines the palaeoinlets were synchronously overgrown by mires. These processes could be strengthened (or intensified) by tectonic processes: glacioisostatic rise of river flanks, and a "tool bend" along a line "Mironov Mountain - Kud'mozero Nikolsky Island" (Fig. 4B). 
After that, in the depressions of the hummocky moraines peat accumulation have been continuing on the marine clays and oxbow peat layer within the ND river valley had been buried by marine deposits during the further Folas and other transgressions.

\section{Folas transgressive - regressive phase (Bore- al/Atlantic): $7710-5745$ cal BC}

We have no chronological data for this period, only boundary dates $8610 \pm 60$ BP $(7710-7570$ BC) and $6920 \pm 40$ BP (5840-5745 BC) bracketing its lower and upper chronological limits (Fig. 6, Table 1).

Morphological data can tell us that during the transgressive period a palaeocliff had been forming in the moraines of Mironov Mountain and southwards the modern terrace, and on the Nikolskiy Island, while peat ac- cumulation had been proceeding in the mires on their surfaces. We suppose that these cliffs could start forming during the Portlandia-Littorina phases, and during the Folas they almost reached their modern pattern.

\section{Lagoon stage}

Tapes-Trivia phase (middle - late Atlantic): 5745-2490 cal BC

This was a long (ca. $\left.6900-4000{ }^{14} \mathrm{C} \mathrm{BP}\right)$ period of higher water stand and/or weak oscillation of sea level.

Since ca. 6900 BP, between Rikasiha site and Nikolskiy Island (Fig. 2A), a series of shallow lagoons with saltish water existed within the area of the modern ND mouth. This is confirmed by dates and composition of deposits in appropriate sections (Fig. 5). Probably, a

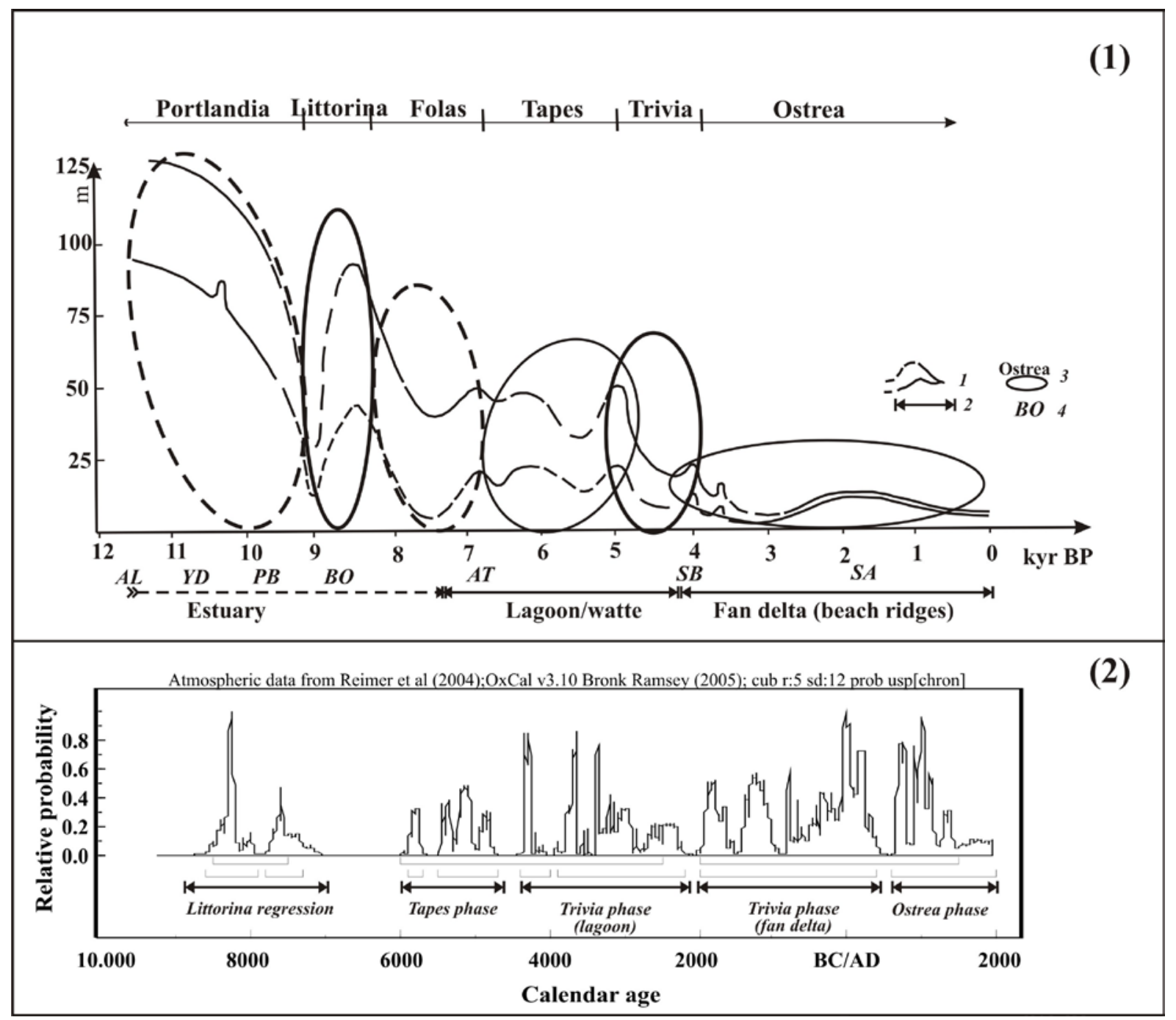

Fig. 6. (1). Changes of the shoreline level of the Barents and White Seas (Kola peninsula) (after Koshechkin, 1979) during the Last Glacial and the Holocene; completed by N.E. Zaretskaya. 1 - the highest and the lowest positions of ancient shorelines; 2 - stages of ND mouth evolution; 3 phases of ND mouth evolution; 4 - Holocene climatic phases. (2). Sum probability distribution of calibrated radiocarbon dates, correlated with stages and phases of the ND delta evolution. 
consequent series of subsynchronous lagoons, and then huge tidal marshes existed in this area, with sand bars or islands between them, and the main discharge of the North Dvina passed through the modern Korabelny arm (Fig. 2A). Actually, lagoons can "survive" within very limited environmental conditions concerning water depth and salinity and time of their development: the increase of water salinity can cause changes into marine conditions, and the decrease of water level - overgrowing and paludification. That is why the firs "stop" or interruption of lagoon development took place ca. 5400 BP (4330 cal $\mathrm{BC}$ ), ending the Tapes stage (sedimentary record and dates, Figs. 5, 6B), and the last and final - ca. 4000-3800 BP (2840-2290 cal BC) (Trivia stage), when the first beach ridge formed within the ND mouth and screened off the lagoons from the White sea.

\section{Fan delta stage}

\section{Trivia-Ostrea phase (end of Atlantic - Subatlantic): 3780 cal BC-present time}

Toward the beginning of Trivia phase (ca. $5000{ }^{14} \mathrm{C}$ $\mathrm{BP}$ ) to the west from the modern ND mouth a vast open marine harbor formed, southerly bounded by Mironov Mountain cliff. This was a time of the ocean level rise stabilization and decrease of the glacioisostatic uplift rate (Koshechkin, 1979; Kaplin and Selivanov, 1999). Within the western part of the study area it manifested in formation of a fan delta (or advanced delta) composed of series of beach ridges (Fig. 2A).

Beach ridges are located in the western part of the modern delta and are characterized by different morphology, dimensions and direction, and generally had been forming since ca. 5000 BP till now. The oldest date was obtained within the Churka unit (Fig. 2A) on the bottom peat between the moraine scarp (palaeocliff) and the first beach ridge and is $4950 \pm 50$ (GIN-14159, 3780-3660 cal $\mathrm{BC})$ and seaward the bottom peat date behind it is $4570 \pm 30$ (GIN-13381, 3370-3330 cal BC), bracketing its age within this short time span (3520-3370 cal BC). This oldest beach ridge is a huge accumulative sand body (width $=100 \mathrm{~m}, 1=3-4 \mathrm{~km}$ ), and is strongly modified by human activity since middle Neolithic till now (Fig. 2A). A huge and long-term Neolithic settlement has been found on this sand body: our reconstructions show that the shoreline existed here quite a long time (3370-1770 cal BC) allowing ancient people to accustom to living at the sea shore, fishing and hunting marine mammals. Since 3530 40 BP (GIN-14014, 1930-1770 cal BC) further consecutive beach ridges have been forming, breaking off the possibilities of seaside occupation by ancient tribes.

Other beach ridges had been formed further later: the next one is dated back at $3530 \pm 40 \mathrm{BP}$ (GIN-14014, 1930$1770 \mathrm{cal} \mathrm{BC}$ ). That is, at the Churka site a time gap in beach ridge formation took place between $4570 \pm 30$ (3370-3330 cal BC) and 3530 \pm 40 BP (1930-1770 cal
$\mathrm{BC}$ ), probably pointing out the Trivia-Ostrea stages transition (Fig. 6).

The next beach ridge series are related to LiasominUglomin Island system which represented earlier a monolithic body. These are sandy sub-parallel ridges alternating with shallow lagoons while advancing into the White Sea (Fig. 2A), now overgrown by mires. The height of the ridges is increasing seawards, from older to younger ones. The youngest beach ridge is dated back at $1080 \pm 40$ BP (GIN-14013, 890-1020 cal AD).

Later on, ca. $3530 \pm 40$ BP (1930-1770 cal BC), beach ridges continued to form in the Churka site and westwards, and since then went synchronously with Liasomin-Uglomin system. Rates of seaward advance of Churka terrace were lower than those of island system (Fig. 2B), and heights and widths of Churka ridges decreased seawards: the most debris that was being discharged by North Dvina river into the White Sea first deposited further west, but later on, while the LiasominUglomin Islands were growing, it was captured by this system.

The advance of the ND delta into the White Sea is still in progress due to the high rate of sediment discharge - 10.1 milion of tons per year (Sokolov, 1952), but its course has been changed by human influence.

\section{CONCLUSIONS}

1) During the Holocene, the North Dvina river mouth had been transforming from the huge and deep estuary through lagoons and tidal marshes into the lobeshaped fan delta with many arms and islands.

2) Occurrence of buried organic sediments at the depth of $17.5 \mathrm{~m}$ and other facts which earlier were considered as evidence of the tectonic subsidence of the delta, could be explained by deep erosional cut in the early Holocene.

3) Estuary (erosional) stage of the ND river mouth development lasted from the early Holocene till ca. $5745 \mathrm{cal} \mathrm{BC}$, and was interrupted by a short accumulation period (8470-7310 cal BC). This resulted in the development of ingression inlets, huge moraine scarp formation and "canyon-type" deep river bed incision.

4) Lagoon (tidal marsh) stage lasted from ca. 5745 till $2290 \mathrm{cal} \mathrm{BC}$, and resulted in the start of modern delta framework formation and accumulation of tidalmarsh deposits. This stage had been interrupted by beach ridges formation which closed water interaction of lagoons with the White Sea basin.

5) Delta stage started about $3780 \mathrm{cal} \mathrm{BC}$ and continues until present times. It resulted in formation of a proper delta (fan delta) and the beach ridges sequence at its western side. On the first western beach ridge, a large Late Neolithic settlement of marine huntergatherers existed between ca. 3370 and 1770 cal BC. 


\section{ACKNOWLEDGEMENTS}

We thank Alexei Belichenko, archaeologist from the Pomor State University in Arkhangelsk, who invited the authors to start their work in this area, and Alexei Edovin from the Arkhangelsk State Museum, for his kind help. We greatly appreciate the results of plant macrofossil analysis performed by Olga Uspenskaya, a unique specialist in this scientific area.

This research became possible due to funding of Russian Foundation for Basic Researches; grant \# 08-0500092.

\section{REFERENCES}

Avenarius IG, 2004. Morphostructure of the White Sea region. Geomorphology 3: 48-56 (in Russian).

Baranovskaya OF, Pleshivtseva ES and Travina MA, 1977. Stratigraphy of the marine deposits of the Late Pleistocene and Holocene of the Lower stream of the North Dvina. In: Biske GS. ed., Stratigraphy and Palaeogeography of the Quaternary of the European North of the USSR, Petrozavodsk: 111-118 (in Russian).

Bohncke S and Simakova AN, 2008. Methods to interpret spore-pollen assemblages. In: AK Markova, T van Kolsfchoten, S Bohncke, PA Kosintsev, J Mol, AYu Puzachenko, AN Simakova, NG Smirnov, A Verpoorte and IV Golovachev, eds., Evolution of European Ecosystems during Pleistocene-Holocene Transition (24-8 kyr $B P$ ). Moscow, KMK: 27-29 (in Russian).

Bradshaw RHW, Holmqvist BH, Cowling SA and Sykes MT, 2000. The effects of climate changes on the distribution and management of Picea abies in southern Scandinavia. Cannadian Joournal of Forest Research 30(12): 1992-1998, DOI 10.1139/cjfr-30-12-1992.

Bronk Ramsey C, 1995. Radiocarbon Calibration and Analysis of Stratigraphy: The OxCal Program. Radiocarbon 37(2): 425-430.

Bronk Ramsey C, 2000. Comment on 'The Use of Bayesian Statistics for $14 \mathrm{C}$ dates of chronologically ordered samples: a critical analysis'. Radiocarbon 42(2): 199-202.

Bratuschak YuV, 2005. Vegetation changes within the Izhma basin over the Holocene. In: Proceedings of the IV Russian Quaternary Symposium, Syktyvkar: 48-50 (in Russian).

Demidov IN, Houmark-Nielsen M, Kjaer KH and E Larsen, 2006. The last Scandinavian Ice Sheet in northwestern Russia: ice flow patterns and decay dynamics. Boreas 35: 1-19.

Kaplin PA and Selivanov AO, 1999. Changes of Russian Seas' level and shore evolution: past, present and future. Moscow, GEOS: 298 pp. (in Russian).

Koshechkin BI, Devyatova, EI, Kagan LYa and Punning YaM, 1977. Lateglacial marine transgression in Onezhskoe Belomorie, Onega Bay of White Sea. In: Biske GS, ed., Quaternary Stratigraphy and Palaeogeography of North of European Part of USSR. KFAN SSSR, Petrozavodsk: 5-16 (in Russian).

Koshechkin BI, 1979. Holocene tectonic of the Eastern part of the Baltic shield. Leningrad, "Nauka": 321 pp (in Russian).

Kremenetsky KV, Patyk-Kara NG and Goriachkin SV, 1998. Palynostratigraphy and geochronology of wetland deposits of the Kola peninsula during the Holocene. Stratigraphy. Geological correlations 6-3: 87-96 (in Russian).

Lavrov AS and Potapenko LM, 2005. Neopleistocene of North-East of the Russian Plain. Aerogeologia, Moscow: 222 pp (in Russian).

Lebedeva RM, 1977. Palynological levels of the Holocene Kola peninsula deposits and their absolute chronology. In: Biske GS, ed., Stratigraphy and Palaeogeography of the Quaternary of the European North of the USSR. Petrozavodsk: 34-39 (in Russian).

Lupachev YuV, 1984. Dynamic interaction of marine and river water within the tidal river mouths. Proceedings of GOIN 172: 64-82 (in Russian).

Nikonov AA, 1977. Holocene and modern movements of the Earth crust: geological, geomorphological and seismological problems. Moscow, "Nauka": 236 pp (in Russian).

Pleshivtseva ES, 1977. Changes of paleogeography conditions in NorthDvina lowland at late-postglacial time. Priroda i hozyaistvo Severa 6: 39-47 (in Russian).

Reimer PJ, Baillie MGL, Bard E, Bayliss A, Beck JW, Bertrand C, Blackwell PG, Buck CE, Burr G, Cutler KB, Damon PE, Edwards RL, Fairbanks RG, Friedrich M, Guilderson TP, Hughen KA, Kromer B, McCormac FG, Manning S, Bronk Ramsey C, Reimer RW, Remmele S, Southon JR, Stuiver M, Talamo S, Taylor FW, van der Plicht J and Weyhenmeyer CE, 2004. IntCal04 Terrestrial Radiocarbon Age Calibration, 0-26 cal kyr BP. Radiocarbon 46(3): 1029-1058.

Sokolov AA, 1952. Hydrography of the USSR. Leningrad, Gidrometeoizdat: 356 pp (in Russian).

Topographic map 1:100.000, Arkhangelsk - Patrakeevka, 2002. FGUP "Aerogeodesia", SPb. (in Russian).

Velichko AA, Andreev AA and Klimanov VA, 1994. Vegetation and climate dynamic of the Northern Eurasia in the Late Glacial and Holocene. In: Short-term and rough climatic changes during the last 15000 years. Moscow, IGRAN: 4-6 (in Russian).

Zaretskaya NE, 2006. Palaeoenvironmental history of the southern White Sea shore over the Holocene (North Dvina delta case study). Proceedings of international scientific conference "Primaeval and medieval history of the Russian North", Solovki: 228-231 (in Russian).

Zaretskaya NE, Ponomareva VV, Sulerzhitsky LD and Zhilin MG, 2001. Radiocarbon studies of peat bogs: an investigation of South Kamchatka volcanoes and Upper Volga archeological sites. Radiocarbon 43(1): 323-335.

Zaretskaya NE, Ponomareva VV and Sulerzhitsky LD, 2007. Radiocarbon dating of large Holocene volcanic events within South Kamchatka (Russian Far East). Radiocarbon 49(2): 1065-1078.

Zaretskaya N, Simakova A, Uspenskaya O and Belichenko A, 2008. Geochronological and palaeoecologic studies of large deltas in cold tidal seas (case of the North Dvina, White Sea, Northern Russia). Abstracts of the 33 International Geological Congress, August 6-14.

Zaretskaya N, Simakova A, Shevchenko N and Uspenskaya O, 2009. Holocene geochronology and palaeoenvironmental record over the North Dvina river delta (White Sea, Northern Russia). In: $A P E X-3$ Abstracts and program, Copenhagen, March 31-April 3: 98-100.

Zhivotovskaya AI, 1960. Young tectonic movements over the Southern White Sea shore. Digest of VSEGEI 29: 119-131 (in Russian).

Zykov DS, Kolodiazhny SYu and Baluev AS, 2008. Features of fundament horizontal neotectonic movements in the White Sea area. Bulletin of Moscow Society of Nature Explorers, geological series 83(2): 15-25. 